\title{
EL COMBATE EN LA ROMA REPUBLICANA: UNA APROXIMACIÓN A LAS CARACTERÍSTICAS GENERALES DE LA BATALLA ANTIGUA
}

\author{
The combat in the Roman Republic. an approach to the general characteristics of \\ the Ancient Battle \\ David SIERRA EsTORNÉS \\ Doctorando en el Departamento de Historia Antigua \\ Universidad Nacional de Educación a Distancia (UNED) \\ E-mail: dasierra11@yahoo.es
}

Fecha de recepción: 6-III-2011

Fecha de aceptación: 30-III-2011

RESUMEN: En esta comunicación se pretende realizar una aproximación a los elementos más importantes que definían el desarrollo de una batalla en la Antigüedad, en este caso, dentro del ámbito romano. Se analizaran factores tales como la larga duración de este tipo de enfrentamientos o el escaso número de bajas del ejército vencedor. Este trabajo se basa en el estudio crítico de las fuentes literarias clásicas. Pretendemos aportar un enfoque distinto del combate antiguo, muy alejado del que nos ha transmitido, principalmente, el cine, pero también la literatura. Ambos nos han presentado las batallas como algo caótico y frenético, donde los combatientes se mezclan y donde la lucha se reduce a un duelo individual. Lo cierto es que los textos clásicos nos transmiten todo contrario y nos describen las batallas como una lucha organizada, prolongada y colectiva.

Palabras clave: Batalla antigua, ejército romano republicano, legionario, combate tentativo, autores clásicos.

ABSTRACT: In this paper we will make an approach to the most important elements that defined the development of a battle in Ancient Times, especially in the roman case. We will consider such factors as the length of such confrontations or the low number of casualties from the victorious army. This paper is based on the critical study of classical literary sources. Our goal is to provide a different approach to the Ancient Combat. The movies and the literature have shown us the battles as a chaotic and 
frantic thing, where the combatants are mixed and where the fight comes down to individual duel. The truth is that the classic texts describe the battles as an organized struggle, prolonged and collective.

Keywords: Ancient Battle, Roman Republican Army, Legionary, Tentative Combat, Classical Writers.

\author{
"El hombre no acude al combate en busca de la \\ lucha, sino de la victoria. Hace todo lo que está en su mano \\ para suprimir la primera y asegurarse la segunda".
}

Ardant du Picq ${ }^{2}$ fue uno de los primeros teóricos militares en analizar los principales elementos que intervenían en el desarrollo de una batalla en la Antigüedad. Su estudio se centra en el análisis de las reacciones del soldado, tanto antiguo como moderno, cuando es sometido a la tensión del combate. Para entender las circunstancias que rodeaban una batalla en la época antigua, es básico comprender su experiencia en combate. Si se parte de esta premisa se entenderá mejor lo que suponía la guerra para el hombre de esta época y el motivo por el que esta era algo tan importante para su modo de vida. Por ejemplo, no podemos olvidar que uno de los factores principales que convirtieron a Roma en uno de los grandes imperios de la Historia fueron sus legiones.

Creemos que existe suficiente información para reconstruir lo que podría haber sido el desarrollo de una batalla en la Roma republicana. En primer lugar, podemos acudir a los relatos bélicos de los autores clásicos, que son nuestras fuentes principales para tratar este asunto. En este sentido, el investigador de la historia militar antigua tiene la suerte de que en las obras históricas conservadas, los temas principales son la política y la guerra. Esto hace que en los textos clásicos la información sobre campañas y batallas sea muy abundante, lo que permite al estudioso del tema analizar el comportamiento de la legión republicana en combate y, en menor medida, aproximarse al funcionamiento de otros ejércitos antiguos. No hay que olvidar que varios de los relatos bélicos de esta época derivan de obras redactadas por antiguos oficiales del ejército romano. En el mejor de los casos, los propios textos conservados fueron escritos por autores con experiencia militar $^{3}$. En segundo lugar, tenemos el único tratado completo sobre el

1 J. J. J. ARDANT DU PICQ, Estudios sobre el combate, Madrid, Ministerio de Defensa, 1988. 2 Ardant du Picq (1819-1870) fue un oficial y teórico militar perteneciente al ejército francés. Su única obra fue Estudios sobre el Combate, en ella Ardant du Picq realizaba un análisis de la batalla antigua y moderna, fijándose sobre todo en la psicología y la moral del combatiente. En su libro destacaba la importancia en la guerra de la disciplina y la cohesión interna de las unidades.

3 Tito Livio, Dionisio de Halicarnaso, Plutarco y Apiano, aún no siendo militares, se basaron 
ejército romano que ha llegado hasta nosotros, el Epitoma rei militaris de Vegecio. Se trata de una obra esencial para el estudio de la legión imperial y, en menor medida, para el conocimiento de la legión republicana ${ }^{4}$. El texto de Vegecio es fundamental porque es el último eslabón en el conocimiento de la doctrina militar romana ${ }^{5}$.

Es cierto que las fuentes clásicas no suelen centrar sus relatos bélicos en detalles sobre aspectos concretos del desarrollo de una batalla antigua, como podría ser la táctica de pequeñas unidades, prefiriendo ofrecer un relato general y resumido del combate. Pero casi siempre dan información interesante y concreta — la forma de lucha de uno y otro bando, su armamento, la existencia de relevos dentro de las líneas, la duración del combate, el número de bajas, etc. — que nos permite reconstruir, en cierta medida, como podía haber sido una batalla en esta época. La información que nos transmiten las fuentes literarias es esencial para comprender el funcionamiento estratégico y táctico del ejército romano. Lo único que hay que hacer es analizar en profundidad y sin prejuicios los datos que se encuentran recogidos en los textos clásicos.

Nuestro análisis, en la medida de los datos existentes, intentará realizar una aproximación a las características generales de la batalla campal en la Roma republicana ${ }^{6}$. Para ello, es esencial comenzar explicando brevemente la doctrina táctica de la legión manipular, que fue la estructura militar más utilizada por los romanos durante este período. Hay que tener en cuenta que sería difícil establecer un modelo único de batalla romana, ya que cada una era distinta de la anterior, con sus propias características, determinadas por varios factores (terreno, clima, enemigo, fuerzas propias, etc.). No obstante, es cierto que normalmente en la mayoría de los enfrentamientos las legiones solían desplegarse siguiendo un mismo patrón (triplex acies). Ha sido Santosuosso ${ }^{7}$ quien mejor ha sintetizado la concepción táctica que subyace en el sistema manipular ${ }^{8}$. Según este autor, los romanos, a diferencia

en los escritos de los analistas, la mayoría de los cuales sirvieron como oficiales en el ejército romano. Polibio, Salustio y Frontino ocuparon cargos militares antes de escribir sus obras.

4 En este sentido, Vegecio entre las fuentes que cita para su obra nos menciona a Catón el Censor, el cual escribió un tratado militar titulado De Re Militari.

5 Las fuentes que cita Vegecio (I, 8, 10) en su tratado son: Catón el Censor (234-149 a.C.), Cornelio Celso (c.25 a.C.-50 d.C), Frontino (c. 40-103 d.C.), Paterno (finales s. III d.C.) y las constituciones de Augusto, Trajano y Adriano (siglos I y II d.C.). Ninguno de estos textos nos ha llegado completo y es a través del Epitoma rei militaris como podemos acceder a la información recogida en ellos, de ahí el gran valor de esta obra.

6 Como obra de síntesis sobre la evolución del ejército romano republicano véase J.M. ROLDAN HERVAS, El ejército de la República Romana, Madrid, Ed. Arco Libros, 1996.

7 A., SANTOSUOSSO, Soldiers, citizens and the symbols of war. From Classical Greece to Republican Rome 500-167 BC, Boulder, Westview Press, 1997, p. 54.

8 La legión manipular comienza a desarrollarse a partir del año 406 a.C., fecha en la que 
de los griegos, entendían la batalla no como un proceso continuo en el cual el primer choque era decisivo, sino como un encuentro escalonado, donde se alternaban distintas fases y en el que se daban pausas entre cada una de ellas. Por este motivo, cada uno de los componentes de la legión manipular cumplía un papel determinado. Los velites desorganizaban al enemigo; los hastati se enfrentaban al adversario a distancia y cuerpo a cuerpo, si no lo derrotaban, al menos lo desgastaban; los principes combatían contra un enemigo ya debilitado y, si no lograban vencerlo se replegaban; por último, los triarii protegían la retirada del resto del ejército hacia el campamento?.

Ahora pasaremos a examinar la duración que podría tener una batalla campal. No existía un tiempo estándar, había enfrentamientos que se resolvían en la primera carga, cuando uno de los dos bandos rompía su formación y huía, antes siquiera de haberse producido el contacto con el enemigo ${ }^{10}$. Lo habitual era que en un enfrentamiento en campo abierto el combate se alargara más allá del primer choque. Por ejemplo, la batalla de Pidna (168 a.C.) duró menos de una hora, tiempo que se consideraba inusualmente corto para lo que era normal en un combate de este tipo ${ }^{11}$. Vegecio fijaba la duración de una batalla en dos o tres horas ${ }^{12}$. Además, tenemos datos en las fuentes que nos indican que este tiempo podía ser incluso mayor. Zhmodikov ${ }^{13}$, basándose en los textos clásicos, ha recogido la duración de varias batallas del período que venimos estudiando.

\begin{tabular}{|l|l|l|l|}
\hline Fecha & Lugar & Duración & Fuente \\
\hline 390 ó 387 a. C. & Alia & $\begin{array}{l}\text { Indeterminada. De corta } \\
\text { duración. Resuelta en la } \\
\text { primera carga. }\end{array}$ & LIVIO, V, 38, 6 \\
\hline 343 a. C. & Monte Gauro & $\begin{array}{l}\text { Batalla larga, de duración } \\
\text { desconocida. }\end{array}$ & LIVIO, VII, 33, 13 \\
\hline 336 a. C. & Cales & $\begin{array}{l}\text { Indeterminada. De corta } \\
\text { duración. }\end{array}$ & LIVIO, VIII, 16, 6 \\
\hline
\end{tabular}

se introduce la paga regular (stipendium) en el ejército romano (LIVIO, IV, 59, 11; VIII, 8, 3). De esta forma, el origen de la organización manipular estaría más relacionado con las circunstancias políticas, sociales y económicas del momento, que con factores estrictamente militares.

9 Para otra buena síntesis sobre el funcionamiento táctico de la legión manipular, véase F. CADIOU, "Les guerres en Hispania et l'émergence de la cohorte légionnaire dans l'armée romaine sous la république", Gladius 21:167-182, 2001, pp. 169-170.

10 LIVIO, V, 38, 6; VIII, 16, 6; XXV, 21, 6; XXXVIII, 27, 11.

11 PLUTARCO, Aem., 22.

12 VEGECIO, Epit., III, 9, 2.

13 A.ZHMODIKOV, "Roman Republican heavy infantrymen in battle (fourth-Second centuries BC)", Historia 49: 67-78, 2000, p. 71, n. 34, 36 y 37. 


\begin{tabular}{|c|c|c|c|}
\hline 322 a. C. & Samnio & Cinco horas. & LIVIO VIII, 38, 10 \\
\hline 320 a. C. & Caudio & $\begin{array}{l}\text { Indeterminada. De corta } \\
\text { duración. }\end{array}$ & LIVIO, IX, 13, 2 \\
\hline 305 a. C. & Boviano & $\begin{array}{l}\text { Batalla larga, de duración } \\
\text { desconocida. }\end{array}$ & LIVIO, IX, 44, 11 \\
\hline 298 a. C. & Volaterra & $\begin{array}{l}\text { Batalla larga, que se prolonga } \\
\text { hasta la noche. }\end{array}$ & LIVIO, X, 12, 5 \\
\hline 295 a. C. & Sentino & $\begin{array}{l}\text { Batalla larga, de duración } \\
\text { desconocida. }\end{array}$ & LIVIO X, 29, 8 \\
\hline 217 a. C. & Lago Trasimeno & Tres horas. & LIVIO, XXII, 6, 1 \\
\hline 216 a. C & Cannas & Todo el día. & $\begin{array}{l}\text { APIANO, Hann., } \\
25\end{array}$ \\
\hline 215 a. C. & Hibera & $\begin{array}{l}\text { Indeterminada. De corta } \\
\text { duración. }\end{array}$ & LIVIO, XXIII, 29, 9 \\
\hline 215 a. C. & Carales & Cuatro horas. & LIVIO, XXIII, 40, 9 \\
\hline 214 a. C. & Benevento & Cuatro horas. & LIVIO, XXIV, 15, 3 \\
\hline 214 a. C. & Munda & Cuatro horas. & LIVIO, XXIV, 42, 2 \\
\hline 212 a. C. & Lucania & Más de dos horas. & $\begin{array}{l}\text { LIVIO, XXV, 19, } \\
15\end{array}$ \\
\hline 212 a. C. & Herdónea & $\begin{array}{l}\text { Indeterminada. De corta } \\
\text { duración. }\end{array}$ & LIVIO, XXV, 21, 6 \\
\hline 212 a. C. & Nola & $\begin{array}{l}\text { Indeterminada. De corta } \\
\text { duración. }\end{array}$ & LIVIO, XXV, 41, 6 \\
\hline 210 a. C. & Numistro & $\begin{array}{l}\text { Batalla larga, de duración } \\
\text { desconocida. }\end{array}$ & LIVIO, XXVII, 2, 7 \\
\hline 209 a. C. & Canusio & Más de dos horas. & $\begin{array}{l}\text { LIVIO, XXVII, } \\
12,14\end{array}$ \\
\hline 206 a. C. & llipa & $\begin{array}{l}\text { Desde el amanecer hasta el } \\
\text { atardecer. }\end{array}$ & $\begin{array}{l}\text { APIANO, Iber., } \\
27\end{array}$ \\
\hline 195 a. C. & Ampurias & $\begin{array}{l}\text { Desde el amanecer hasta el } \\
\text { atardecer. }\end{array}$ & $\begin{array}{l}\text { APIANO, Iber., } \\
40\end{array}$ \\
\hline 193 a. C. & Lusitania & Cinco horas. & LIVIO, XXXV, 1, 5 \\
\hline 179 a. C. & Monte Cauno & Seis horas. & LIVIO, XL, 50, 2 \\
\hline
\end{tabular}




\begin{tabular}{|l|l|l|l|}
\hline 173 a. C. & Caristo & Más de tres horas & LIVIO, XLII, 7, 5 \\
\hline 168 a. C. & Pidna & Menos de una hora. & $\begin{array}{l}\text { PLUTARCO, } \\
\text { Aem., 22. }\end{array}$ \\
\hline 141 a. C. & Termancia & Todo el día. & $\begin{array}{l}\text { APIANO, Iber., } \\
77\end{array}$ \\
\hline
\end{tabular}

Tabla 1. Duración de batallas (390-141 a. C.)

A la luz de estos datos, se puede llegar a la conclusión de que la mayoría de combates que los romanos disputaban en campo abierto eran de larga duración. En este sentido, la disposición de la legión manipular en varias líneas, circunstancia que permitía el relevo de las unidades de vanguardia, estaría pensada para una lucha prolongada, ya que no sería necesario intercambiar a los combatientes en un enfrentamiento de corta duración ${ }^{14}$.

A lo mencionado anteriormente, habría que añadir el tiempo que llevaría a las distintas unidades que formaban las legiones salir del campamento, avanzar hacia el campo de batalla y colocarse en la posición asignada por sus oficiales ${ }^{15}$. En definitiva, la larga duración de las batallas que implicaban al ejército romano estaría muy ligada al modo en el que las legiones combatían.

Otro factor de gran importancia a la hora de comprender el desarrollo de una batalla en la Roma republicana era el número de bajas que se producían en este tipo de enfrentamiento. La información que tenemos proviene de las fuentes literarias. El principal problema que se plantea es la credibilidad que podemos dar a las cifras que los autores clásicos nos suministran, ya que en algunos casos se producen contradicciones entre dos fuentes distintas sobre el número de bajas en un mismo enfrentamiento. El ejemplo más conocido es el de la batalla de Metauro (207 a.C.), donde Livio nos dice que murieron ocho mil romanos y cincuenta y siete mil cartagineses ${ }^{16}$ y Polibio, en cambio, nos indica que los muertos fueron dos mil romanos y diez mil púnicos ${ }^{17}$.

Aún así, en relación con estos datos, lo que a nosotros nos interesa resaltar es el número tan escaso de muertos que el ejército ganador sufre en comparación con las bajas del perdedor. Krentz ${ }^{18}$ después de analizar una serie de batallas entre falanges hoplíticas (472-371 a.C.), llega a la conclusión de que la media de muertos en el bando ganador se sitúa en el $5 \%$ de su fuerza total. En el bando derrotado esta cifra se sitúa en el 14\%. Gabriel y

14 A. ZHMODIKOV, op. cit., p. 71.

15 G. DALY, Cannae, London, Routledge, 2002. p. 165.

16 LIVIO, XXVII, 49, 5-8.

17 POLIBIO, XI, 3, 3.

18 P. KRENTZ, “Casualties in hoplite battle”, GRBS 26: 13-21, 1985, p. 19. 
Metz ${ }^{19}$ después de examinar las cifras de bajas de varias batallas antiguas ${ }^{20}$, concluyen que el porcentaje medio de muertos que sufre el ejército derrotado se sitúa en el $37,7 \%$ de su fuerza inicial; en cambio, el ejército vencedor pierde sólo un 5,5\% del total de sus hombres. En el ejército romano, este último porcentaje es similar y en algunos casos es incluso menor del $5 \%$.

\begin{tabular}{|c|c|c|c|c|c|}
\hline Fecha & Batalla & $\begin{array}{l}\text { Fuerzas } \\
\text { totales }\end{array}$ & Bajas & Porcentaje & Fuente \\
\hline 202 a. C. & Zama & 35.100 & 1.500 muertos & $4,2 \%$ & $\begin{array}{l}\text { APIANO, } \\
\text { Afr., 41; } \\
\text { POLIBIO, } \\
\text { XV, 5, 12; } \\
14.9\end{array}$ \\
\hline 197 a. C & Cinoscéfalos & 26.000 & 700 muertos & $2,6 \%$ & $\begin{array}{l}\text { PLUTARCO, } \\
\text { Flam., 7, 2; } \\
\text { POLIBIO, } \\
\text { XVIII. } 27.6\end{array}$ \\
\hline 190 a. C. & Magnesia & 30.000 & 349 muertos & $1,1 \%$ & $\begin{array}{l}\text { APIIANO, } \\
\text { Syr., 31; } \\
\text { LIVIO, } \\
\text { XXXVII 44,2 }\end{array}$ \\
\hline 168 a. C. & Pidna & 30.000 & 100 muertos & $0,3 \%$ & $\begin{array}{l}\text { LIVIO, XLIV, } \\
42,8\end{array}$ \\
\hline 101 a. C. & Vercellae & 52.300 & 300 muertos & $0,5 \%$ & $\begin{array}{l}\text { PLUTARCO, } \\
\text { Mar., 25, 6; } \\
\text { FLORO, I, } \\
38.14\end{array}$ \\
\hline 48 a. C. & Farsalo & 22.000 & $\begin{array}{l}\text { 230/1.200 } \\
\text { muertos }\end{array}$ & $1,0 / 5,4 \%$ & $\begin{array}{l}\text { CESAR, } \\
\text { B.Civ, III, } \\
89,2 ; 99, \\
\text { 1; APIANO, } \\
\text { B.Civ. 2.82 }\end{array}$ \\
\hline 45 a. C. & Munda & 40.000 & $\begin{array}{l}1.500(1.000 \\
\text { muertos y } 500 \\
\text { heridos) }\end{array}$ & $3,7 \%$ & $\begin{array}{l}\text { CESAR, } \\
\text { B.Hisp., } \\
30-31\end{array}$ \\
\hline
\end{tabular}

Tabla 2. Victorias romanas. Porcentaje de bajas del ejército vencedor.

Al número de muertos que nos presentan las fuentes habría que sumar el de los heridos ${ }^{21}$. Los autores clásicos no suelen ser explícitos en este aspecto, de hecho la mayoría de las veces no mencionan la cantidad de

19 R. GABRIEL AND K.METZ, From Sumer to Rome: the military capabilities of ancient armies, London, ABC-CLIO, 1991, p. 86.

20 Su estudio abarca desde la época sumeria hasta Roma. A la hora de examinar los datos que nos proporcionan las fuentes sobre las batallas en la Antigüedad se centran principalmente en las libradas por los griegos, los macedonios y los romanos.

21 P. A. G. SABIN, “The face of Roman battle”, JRS 90: 1-17, 2000, pp. 5-6. 
heridos que se han producido en una batalla, sino que prefieren centrase en las cifras de muertos y prisioneros. La mayoría de los heridos los sufría el ejército vencido tras romper su formación de combate y emprender la huida. Normalmente, los porcentajes de muertos y heridos para ambos bandos serían bajos, mientras ambos ejércitos mantuvieran sus formaciones de combate. Engels ${ }^{22}$, en su estudio sobre el ejército de Alejandro Magno, calcula que en las batallas campales los macedonios sufrieron cinco heridos por cada muerto.

En relación con las cifras de muertos que las fuentes clásicas nos detallan, puede que en algunos casos estos datos hayan sido exagerados por los autores antiguos con el fin de resaltar una victoria, pero lo cierto es que, aún así, son siempre cifras muy bajas. Cuando una fuente habla de las pérdidas humanas de un enemigo extranjero, probablemente las cifras sean exageradas por varios motivos, pero sobre todo por la dificultad de cuantificarlas. En cambio, sabemos que en el ejército romano todo se controlaba, fiscalizaba y registraba ${ }^{23}$ (las listas de reclutamiento, los días de servicio, las recompensas, los castigos, las pagas, el reparto del botín, etc.). Nada más fácil para saber el número de bajas, que contar cuantos legionarios había en una unidad al principio de una batalla y cuantos quedaban después del combate. Posteriormente, estos datos podrían haber sido recogidos en algún tipo de registro oficial. Por eso, este tipo de cifras pueden ser más fiables. Sabemos que después de una victoria, los generales romanos enviaban cartas laureadas al senado informándole del desarrollo y del resultado de la batalla que había tenido lugar ${ }^{24}$.

En Grecia, en ciudades como Atenas, los nombres de los muertos en combate eran normalmente inscriptos en piedra, habiéndose conservado algunos fragmentos de estas listas. La información estaba disponible para cualquier habitante de Atenas y sería lógico pensar que los historiadores consultarían este tipo de documentos ${ }^{25}$. Por todo esto, no nos parece descabellado pensar que los historiadores antiguos, a la hora de elaborar sus relatos bélicos y más concretamente al cuantificar las bajas, pudieron acceder a estos datos de forma directa o indirecta. En el caso romano, siguiendo con la idea, anteriormente expuesta, sobre las cartas enviadas por los generales al senado, tenemos un magnifico ejemplo de este proceso, con lo ocurrido después de la victoria romana en Metauro (207 a. C.). Los cónsules, tras la

22 D. W. ENGELS, Alexander the Great and the logistics of the Macedonian army, Berkeley, University of California Press, 1978, p. 151.

23 Prueba de ello, son las siguientes obras, que aunque se ocupan del ejército imperial, aportan información, basada en restos arqueológicos, sobre la organización administrativa de las legiones. Véanse A. R. BIRLEY, Garrison life at Vindolanda, Stroud, Tempus, 2002. R. O. FINK, Roman military records on papyrus, Cleveland, CWRU Press, 1971.

24 DIONISIO, VI, 13, 3; LIVIO, V, 28, 13; VIII, 30, 10.

25 P. KRENTZ, op. cit., pp. 13-14. 
batalla, redactan una carta contando lo acontecido en la lucha. La misiva es llevada a Roma por tres emisarios. Cuando llegan a la ciudad, la carta primero es leída al senado y después al pueblo. Más tarde, uno de los mensajeros realiza una exposición pública más detallada de lo acontecido durante el combate $^{26}$. Teniendo presente lo que hemos explicado, es lógico pensar que el número de bajas sufridas por los romanos en una batalla, apareciera recogido en estas cartas. Posteriormente, está información se registraría en algún archivo oficial, como por ejemplo, los annales maximi, que entre otros acontecimientos, recogían los ocurridos en la guerra ${ }^{27}$.

A modo de resumen, sobre este tema, podemos decir que en una batalla antigua los dos bandos enfrentados soportaban relativamente pocas bajas antes de que uno de los dos iniciara la huida. El verdadero peligro para el ejército vencido, en especial para los que se encuentran incapacitados por sus heridas, empieza sólo después de que los hombres que lo componen vuelvan la espalda y huyan. Incluso en los combates más prolongados que libraron las legiones, donde ninguno de los contendientes iniciaba la huida y donde la lucha concluía sin un vencedor claro, las bajas eran escasas en ambos ejércitos ${ }^{28}$. Es una paradoja, pero cuando el soldado cree que su salvación está en correr y huir del enemigo es cuando en mayor peligro se encuentra, al no tener ya la protección que sus compañeros le otorgaban dentro de la formación. En la Antigüedad el miedo es el verdadero asesino en el campo de batalla. Los hombres en combate controlan su reacción instintiva de huir ante el peligro mediante los dictados de su inteligencia, que le indica que la mejor manera de sobrevivir en una batalla es combatiendo dentro de un grupo y no de forma individual. Los soldados de todas las épocas, amparándose en la protección del grupo, realizan acciones que no ejecutarían en solitario ${ }^{29}$.

Otro factor que consideramos importante en nuestra aproximación a la batalla antigua es el de la posible confusión que rodeaba a los soldados de ambos ejércitos en medio del combate ${ }^{30}$. Estos eran conscientes sólo de lo que ocurría a su alrededor y de lo que pasaba en las proximidades de su unidad, no teniendo conocimiento de lo que en ese momento sucedía en el resto del campo de batalla ${ }^{31}$. La solución que los romanos adoptaron para resolver este problema fue la de dividir a la legión en unidades más pequeñas, que en el caso de la época que estudiamos estas unidades serían los manípulos y las centurias. La otra solución fue la de dotar a cada centuria

26 LIVIO, XXVII, 51, 2-6.

27 SERVIO, Ad Verg. Aen. 1, 373.

28 P. A. G. SABIN, op. cit., p. 6.

29 R. GABRIEL, The Ancient World, London, Greenwood Publishing Group, 2007, p.131.

30 A. D. LEE, "Morale and the Roman Experience of Battle", in A.B.Lloyd (ed.), Battle in Antiquity, London, 1996, p. 201.

31 TUCÍDIDES, VII, 44. 
de un estandarte (signum) o de un pendón (vexillum); de esta forma, cada legionario podía saber en todo momento donde estaba su unidad ${ }^{32}$. La primera referencia al uso de los estandartes por parte de los romanos, se sitúa en los tiempos de Rómulo. Al frente de cada unidad del ejército de este rey, marchaba un hombre enarbolando una lanza coronada con un manojo de heno (manipulus) ${ }^{33}$

Las causas de esta confusión eran varias. En primer lugar, sabiendo que la mayoría de las campañas militares tenían lugar en primavera y verano, el polvo que se levantaba en el campo de batalla sería un elemento que aumentaría la confusión ${ }^{34}$. Su causa estaría en el movimiento de la caballería y la infantería de ambos ejércitos ${ }^{35}$.

En segundo lugar, otro de los elementos que potenciaba la sensación de confusión del soldado antiguo era la profundidad de las formaciones que adoptaban los ejércitos en combate. En el caso romano, el fondo de sus unidades oscilaría entre los tres y los diez hombres ${ }^{36}$. Los legionarios de la primera fila tendrían una percepción relativamente buena de lo que estaba sucediendo en comparación con el resto de sus compañeros, situados detrás de ellos. La visión de los hombres de las siguientes líneas estaría limitada por el espacio cubierto por los compañeros ubicados delante de ellos, que obstruirían su visión de lo que sucedía en vanguardia. Los legionarios colocados en las últimas filas no verían prácticamente nada de lo que estaba ocurriendo en la primera línea de combate. Su información sobre como se estaba desarrollando la batalla se basaría en los movimientos de su unidad; si avanzaba es que las cosas iban bien, si retrocedía era un signo de que algo iba mal. Como cualquier soldado de todas las épocas, el combatiente antiguo estaba rodeado por la incertidumbre, no teniendo una percepción clara de lo que sucedía, no ya únicamente en el resto del campo de batalla, sino dos o tres filas por delante de él ${ }^{37}$.

En tercer lugar, el ruido era otro de los elementos que potenciaban una sensación de confusión en el legionario romano. Al sonido de las órdenes de los oficiales, se le unirían los ruidos provocados de forma accidental; como el relinchar de los caballos, o el sonido producido por el roce de las armas y las armaduras de miles de soldados avanzando hacia el enemigo. También produciría un ruido ensordecedor el lanzamiento de jabalinas y flechas, junto

32 VEGECIO, II, 13, 2-3.

33 PLUTARCO, Rom., 8, 7; SERVIO, Ad Verg. Aen. 11, 870.

34 G. DALY, op. cit., p. 166.

35 POLIBIO, V, 85, 12; ONASANDRO, VI, 8.

36 Número de combatientes de fondo: tres (JOSEFO, B. Jud., II, 113); cuatro (APIANO, Gall., 1, 1); entre tres y seis (VEGECIO, Epit., III, 15); ocho (ARRIANO, Ekt., 15); diez (FRONTINO, Strat., II, 3, 22).

37 C. CARLTON, Going to the Wars, London, Routledge, 1992, p. 133. 
con el choque de las espadas, las lanzas y los escudos de los combatientes ${ }^{38}$. Otros sonidos involuntarios serían los originados por los gruñidos y gemidos emitidos por los soldados mientras combatían, pero sobre todo los gritos y los lamentos procedentes de los hombres y caballos heridos ${ }^{39}$.

En una batalla también podían escucharse sonidos generados con la intención de atemorizar al enemigo ${ }^{40}$. Un ejemplo típico de esto se producía cuando los combatientes avanzaban al encuentro de sus rivales. El ejército republicano marchaba hacia el enemigo con gran estrépito, con los legionarios gritando y golpeando sus escudos con las armas ${ }^{41}$. Los celtas, para asustar al enemigo, usaban el carnyx, un instrumento de viento en forma de cuerno $^{42}$. Además de los celtas, muchos pueblos de la Antigüedad utilizaban instrumentos similares para amedrentar al enemigo, como los partos ${ }^{43}$. Estos habían llegado a la conclusión que la mejor forma de producir miedo en el adversario era atacando su sentido del oído. De esta forma, los partos empezaban ganando la batalla psicológica ${ }^{44}$.

En estas circunstancias, para evitar los posibles problemas de transmisión de órdenes que se podían generar entre los oficiales y sus subordinados, los ejércitos antiguos usaban distintos tipos de señales. En primer lugar, estaba la comunicación verbal, pero muchas veces esta no era posible debido al ruido ambiental, provocado por la multitud de hombres y caballos que se enfrentaban en el campo de batalla. En segundo lugar, tendríamos las señales visuales como los signa y vexilla, pero a veces estas eran ineficaces debido a la niebla, el polvo, la nieve, la lluvia o la presencia de obstáculos como árboles o accidentes del terreno. Para evitar los problemas visuales comentados, se usaban las señales sonoras producidas por instrumentos musicales como la trompeta ${ }^{45}$. Ejemplo de este tipo de instrumentos eran el cornu, la tuba y la bucina romanas. Por último, no podemos olvidar la importancia de la caballería, que gracias a su movilidad y rapidez, era un medio eficaz para transmitir órdenes, circunstancia que se mantuvo en los campos de batalla hasta principios del siglo XX.

A la hora de analizar como serían los enfrentamientos de la infantería pesada romana con sus enemigos, la mayoría de los expertos coinciden en defender un tipo de lucha basada en el choque entre dos líneas formadas, en la que sólo los hombres de las filas delanteras intervienen directamente en el

38 AMIANO MARCELINO, XVI, 12, 46.

39 AMIANO MARCELINO, XVI, 12, 37.

40 POLIBIO, II, 29, 5-7.

41 POLIBIO, XV, 12, 7-9.

42 G. DALY, op. cit., p. 169.

43 A. K GOLDSWORTHY, op. cit., p. 195.

44 PLUTARCO, Crass., 23.

45 ARRIANO, Tact., 27. 
combate ${ }^{46}$. Nos surge la duda de cuántos de estos guerreros participaban activamente en la lucha. Dar respuesta a esta cuestión nos ayudaría a entender mejor como era el combate antiguo.

Ha sido Goldsworthy ${ }^{47}$ quien más ha estudiado este tema, llegando a la conclusión de que la mayor parte de los combatientes situados en vanguardia luchan más con la intención de mantenerse con vida que con el propósito de eliminar al enemigo. Estos hombres practicarían un tipo de lucha de carácter tentativo, donde el objetivo sería herir al adversario, pero sin arriesgar la propia integridad física; porque lo cierto es que para cualquier soldado, tanto del presente como del pasado, lo primero es sobrevivir y lo segundo ganar la batalla en la que participa. En todos los ejércitos hay unos pocos hombres que destacan del resto porque pelean de forma más agresiva y arriesgada, por eso mismo en las legiones se recompensaba a los hombres que asumían riesgos y combatían con valor ${ }^{48}$, siempre que lo hicieran siguiendo las órdenes de sus oficiales y se mantuvieran dentro de su unidad. En caso contrario, estos hombres podían ser duramente castigados por contravenir las órdenes o por abandonar la formación ${ }^{49}$.

Los escasos datos que nos suministran los autores clásicos, en relación con este tema van en la misma línea que venimos comentando, en una batalla igualada el resultado se decidía por la acción de unos pocos hombres ${ }^{50}$. En este sentido, es bastante significativo el siguiente pasaje:

\section{Victoria enim perpaucos fieri consueuit. Tantum est,} ut electi a duce sapientissimo in his locis, in quibus ratio et utilitas postulat, ordinentur ${ }^{1}$.

Otro aspecto del combate legionario que consideramos de gran importancia, es la duración de los choques de los manípulos romanos contra las unidades enemigas y el tiempo que transcurría en la consiguiente lucha cuerpo a cuerpo. Se ha estimado que el tiempo máximo que un hombre podía combatir cuerpo a cuerpo en una batalla antigua, antes de quedar exhausto, era de quince minutos ${ }^{52}$. Este tipo de lucha, con la espada y el

46 POLIBIO, XVIII, 30, 10-11; P. A. G. SABIN, op. cit., p. 10.

47 A. K GOLDSWORTHY, The Roman Army at War, 100 BC-AD 200, Oxford, Oxford University Press, 1996, pp. 219-222.

48 POLIBIO, VI, 39.

49 DIODORO, XII, 64, 3; LIVIO, VIII, 7,1-22; SALUSTIO, Cat., 9, 4; CÉSAR, B.Gal., VII, 52.

50 TÁCITO, Ann., XIV, 36, 2; LIVIO, 24, 15, 4-5.

51 VEGECIO, Epit., III, 20, 28: "La victoria normalmente se consigue gracias a pocos soldados. Únicamente deben ser elegidos por un comandante inteligente y ser colocados en las posiciones que dictan el sentido común y la pragmática" (Trad. D. Paniagua).

52 J. F. C. FULLER, Julius Caesar: man, soldier and tyrant, New York, Rutgers University Press, 
escudo, no podía haber sido muy larga debido al desgaste físico y a la tensión emocional que conllevaba. De esta forma, tras un breve período de combate cuerpo a cuerpo, ambas formaciones enfrentadas se habrían separado, dejando entre ellas una distancia de seguridad de unos pocos metros. Este sería el momento en el cual los legionarios descansarían, se retiraría a los heridos y se relevaría a las unidades agotadas por otras frescas provenientes de la segunda línea ${ }^{53}$.

Estos instantes de pausa y de tregua entre ambos contendientes serían el estado natural de estos enfrentamientos ${ }^{54}$. Es decir, la mayor parte de una batalla legionaria transcurriría con ambos bandos separados por cierta distancia, donde la norma era el intercambio de proyectiles y la excepción el combate cuerpo a cuerpo. En este contexto, los ejércitos más disciplinados y sobre todo mejor dirigidos, como era el caso de los romanos con sus legiones y centuriones ${ }^{55}$, desde esta distancia de seguridad avanzarían al unísono contra el enemigo. En cambio, las tropas menos disciplinadas, como sería el caso de los galos, realizarían repetidas cargas descoordinadas entre sí lideradas por los guerreros más audaces. Después de un rápido intercambio de estocadas y golpes con el contrario, uno de los dos bandos - la formación que en el choque se hubiera llevado la peor parte - se retiraría hasta volver a establecer la distancia de seguridad inicial, momento en el que se fijaría involuntariamente un período de tregua entre ambas fuerzas. Tras esta fase de descanso se volvería nuevamente al cuerpo a cuerpo ${ }^{56}$. De esta forma en una batalla se producía una sucesión de avances y retrocesos localizados a lo largo de la línea de combate, de forma que esta unas veces se apretaba y otras se curvaba hacia delante ${ }^{57}$.

En una batalla, muchas unidades cargaban, haciendo recular al enemigo unos pocos metros. La fuerza que retrocedía lo hacía después de un breve combate y tras haberse llevado la peor parte en el choque. Los vencedores de estos enfrentamientos locales sufrían pocas bajas, que se irían acumulando con cada nuevo asalto ${ }^{58}$. La lucha estaba llena de largos períodos

1965, p. 91

53 A. K GOLDSWORTHY, op. cit., pp. 224-225.

54 F. QUESADA SANZ, "El legionario romano en la época de las Guerras Púnicas. Formas de combate individual, táctica de pequeñas unidades e influencias hispanas", Espacio, tiempo y forma. Serie II, Historia antigua, No 16: 163-196, 2003, p. 185.

55 Sobre la disciplina romana véase J. E. LENDON, Soldados y fantasmas, Barcelona, Ed. Ariel, 2006, pp. 226- 229.

56 P. A. G. SABIN, "The mechanics of battle in the Second Punic War", in T.J.Cornell, N.B. Rankov, and P.Sabin (eds), The Second Punic War: A Reappraisal, London, Institute of Classical Studies, 1996, p. 72.

57 PLUTARCO, Aem., 20, 7-9; PLUTARCO, Pyrrh., 17, 2.

58 A. K GOLDSWORTHY, op. cit., p. 227. 
de inactividad, cuando ninguno de los bandos estaba dispuesto a avanzar y ambas fuerzas permanecían separadas por una cierta distancia de seguridad mientras se lanzaban jabalinas. El resto del tiempo se gastaba en breves combates cuerpo a cuerpo. Debido a que los suministros de proyectiles son limitados y que la mayoría de estas armas arrojadizas son interceptadas por los largos escudos de la infantería pesada, es posible pensar que estas batallas continuarían durante horas y las bajas serían pocas, tal y como lo atestiguan las fuentes ${ }^{59}$.

A modo de conclusión queremos terminar nuestra aproximación al combate en la Roma republicana con dos textos que sintetizan bien como podría haber sido una batalla antigua. El primero se sitúa durante el enfrentamiento de Filipos (42 a. C.):

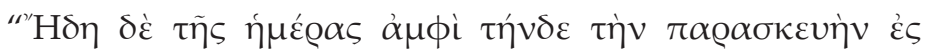

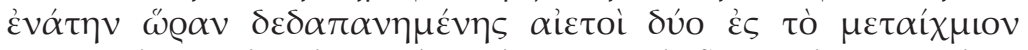

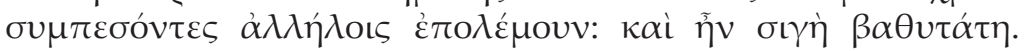

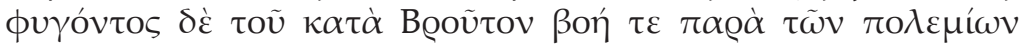

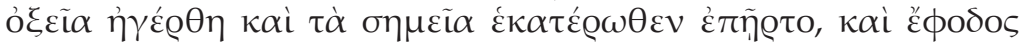

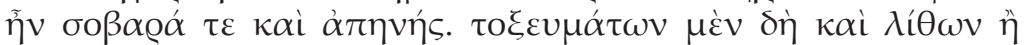

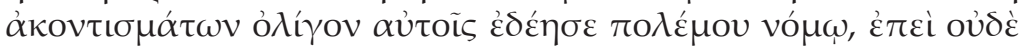

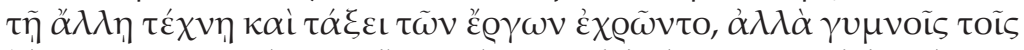

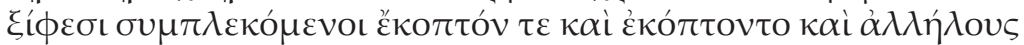

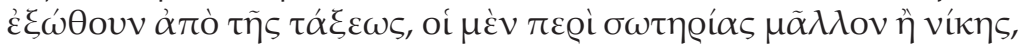

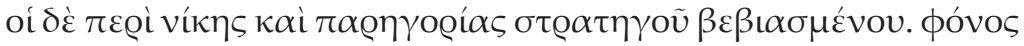

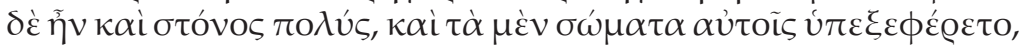

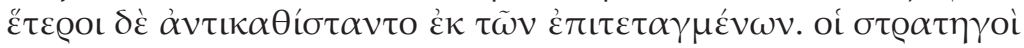

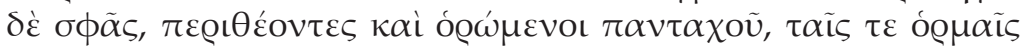

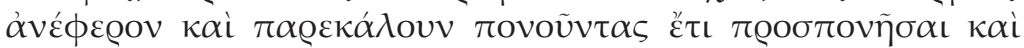

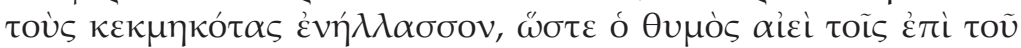
$\mu \varepsilon \tau \omega \dot{\pi} \pi \mathrm{ov}$ kaเvòs $\tilde{\eta} \mathrm{v}^{\prime \prime 60}$.

En este texto podemos apreciar varios datos sumamente interesantes. En primer lugar, se nos indica como el empleo de armas arrojadizas era algo muy común en las batallas de la Antigüedad. Pero en este caso, por el ardor combativo de ambos ejércitos, se había prescindido de ellas. En segundo

59 P. A. G. SABIN, op. cit., p. 72.

60 APIANO, B. Civ., IV, 128: "La carga fue soberbia y terrible. Poca necesidad habia de flechas, piedras o jabalinas, como era costumbre en la guerra, puesto que ni siquiera se servian de las diversas estrategias y posicionamientos usuales en los combates, sino que, en combate cuerpo a cuerpo y con las espadas desnudas, asestaban y recibian los golpes mortales y trataban mutuamente de expulsarse de la formación [...] La carnicería y los gritos de dolor eran enormes. Los cuerpos de los que caían eran retirados del campo de batalla y otros ocupaban su lugar procedentes de las tropas de reserva. Los generales, mientras recorrian sus filas e inspeccionaban todo, los animaban por su celo, y exhortaban a los combatientes a persistir en la lucha y relevaban a los que estaban exhaustos, de tal forma que siempre babia un ardor renovado en el frente" (Trad. A. Sancho). 
lugar, los legionarios de ambos contingentes peleaban con sus espadas con el objetivo de herir y expulsar a su adversario de la posición que ocupaba. En tercer lugar, los gritos de los heridos hacían que la confusión sonora fuera aún mayor de lo que era normalmente en el campo de batalla. En cuarto lugar, este texto nos describe la correcta actuación de los comandantes de ambos ejércitos, que hacían lo que todo buen general debe hacer en una batalla: moverse entre las filas de sus hombres, observando y animando a sus soldados ${ }^{61}$. Pero sobre todo, ambos líderes hacían algo esencial para el éxito de sus tropas, se encargaban de relevar a los legionarios agotados por fuerzas de refresco provenientes de las unidades de reserva, para que así el empuje de sus respectivas vanguardias no decayera.

El segundo texto se sitúa en la batalla de Forum Gallorum (43 a. C). Parece que Apiano, por la cercanía a lo narrado que nos transmite, ha recogido la descripción de un testigo presencial de los hechos, probablemente un alto oficial romano que estaba muy próximo a la línea de combate:

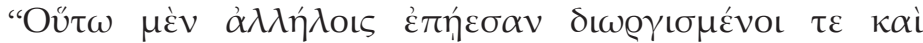

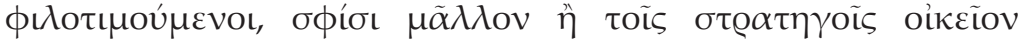

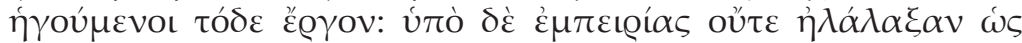

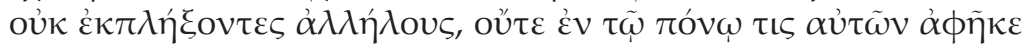

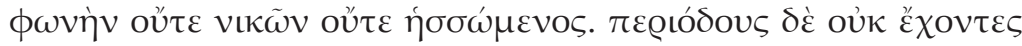

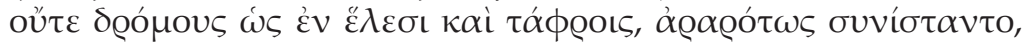

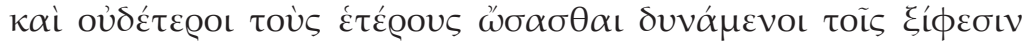

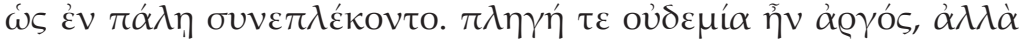

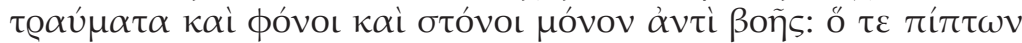

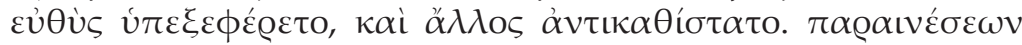

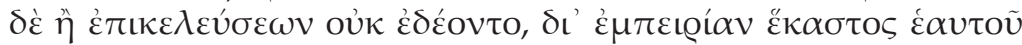

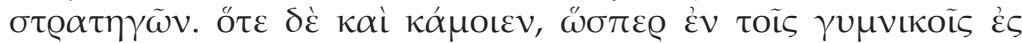

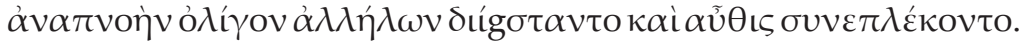

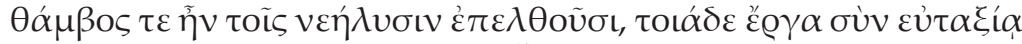

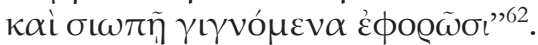

En este pasaje se nos describen detalles interesantes sobre la forma de combatir de las legiones romanas. En primer lugar, vemos como las unidades

61 Lo normal era que el general romano se desplazara, entre las unidades de su ejército, montado a caballo. Situación que le posibilitaba acudir con rapidez a los puntos críticos de la línea de batalla. Además, la altura del caballo permitía al general observar mejor el desarrollo de la lucha, a la vez que era testigo del comportamiento de sus hombres.

62 APIANO, B. Civ., III, 68: "Como no había lugar a evoluciones y cargas por combatir en zona pantanosa y con fosos luchaban codo con codo y al no poder rechazar al adversario se enzarzaban entre sí con las espadas como en una lucha entre atletas. El que caía era retirado al punto y otro ocupaba su lugar. No bacian falta gritos de aliento o animo, la experiencia de cada hombre hacia de si mismo su propio general. Cuando estaban agotados de fatiga, como en los ejercicios gimnásticos, se separaban un poco para tomar respiro y de nuevo se reintegraban a la lucha. El estupor se apoderó de los bisoños, cuando llegaron al contemplar tales luchas realizadas en profundo silencio y orden" (Trad. A. Sancho). 
de ambos ejércitos chocan y como, cuando alguien cae herido, es retirado rápidamente (suponemos que a retaguardia), ocupando otro hombre su lugar (deducimos que proveniente de la segunda fila). Esto parece indicarnos que el atacante, después de herir a su rival, no avanzaría para intentar penetrar en la formación enemiga por el hueco que ha dejado su oponente caído, más bien lo contrario, se mantiene a la expectativa mientras el contrario es retirado y otro ocupa su lugar. En caso contrario, si llegara a introducirse en la unidad adversaria asumiría un gran riesgo, al encontrase rodeado de enemigos que tratarían de matarle. En segundo lugar, se aprecia claramente como ambas formaciones, tras un período de lucha cuerpo a cuerpo, se separan y después de un tiempo de descanso vuelven a chocar. Esto parece confirmarnos lo que comentábamos antes sobre las continuas pausas y las repetidas cargas que se daban en una batalla antigua.

En definitiva, creemos que teniendo como base las fuentes literarias, junto con la investigación comparativa, en circunstancias similares, de otros períodos históricos mejor documentados, es posible llegar a reconstruir el desarrollo general de lo que podía haber sido una batalla en la Antigüedad. 